\title{
Efflux-mediated fluoroquinolone resistance in the multidrug-resistant Pseudomonas aeruginosa clinical isolate PA7: identification of a novel MexS variant involved in upregulation of the mexEF-oprN multidrug efflux operon
}

\section{Yuji Morita*, Junko Tomida and Yoshiaki Kawamura}

Department of Microbiology, School of Pharmacy, Aichi Gakuin University, Nagoya, Japan

\section{Edited by:}

Keith Poole, Queen's University,

Canada

Reviewed by:

Antonio Oliver, Hospital Son Dureta, Spain

Herbert P. Schweizer, Colorado State University, USA

Patrick Plesiat, University of

Franche-Comté, France

*Correspondence:

Yuji Morita, Department of

Microbiology, School of Pharmacy,

Aichi Gakuin University, 1-100

Kusumoto, Chikusa, Nagoya, Aichi 464-8650, Japan

e-mail:yujmor@dpc.agu.ac.jp
The emergence of multidrug-resistant Pseudomonas aeruginosa has become a serious problem in medical settings. $P$. aeruginosa clinical isolate PA7 is resistant to fluoroquinolones, aminoglycosides, and most $\beta$-lactams but not imipenem. In this study, enhanced efflux-mediated fluoroquinolone resistance of PA7 was shown to reflect increased expression of two resistance nodulation cell division (RND) -type multidrug efflux operons, mexEF-oprN and mexXY-oprA. Such a clinical isolate has rarely been reported because MexEF-OprN-overproducing mutants often increase susceptibility to aminoglycosides apparently owing to impairment of the MexXY system. A mutant of PA7 lacking three RND-type multidrug efflux operons (mexAB-oprM, mexEF-oprN, and mexXY-oprA) was susceptible to all anti-pseudomonas agents we tested, supporting an idea that these RND-type multidrug efflux transporters are molecular targets to overcome multidrug resistance in $P$. aeruginosa. mexEF-oprN-upregulation in $P$. aeruginosa PA7 was shown due to a MexS variant harboring the Valine-155 amino acid residue. This is the first genetic evidence shown that a MexS variant causes mexEF-oprN-upregulation in $P$. aeruginosa clinical isolates.

Keywords: Pseudomonas aeruginosa, efflux, mexXY-oprA, mexEF-oprN, mexS

\section{INTRODUCTION}

Pseudomonas aeruginosa is a metabolically versatile bacterium that can cause a wide range of severe opportunistic infections in patients with serious underlying medical conditions (Gellatly and Hancock, 2013). Infections caused by $P$. aeruginosa often are hard to treat; inappropriate chemotherapy readily selects multidrugresistant $P$. aeruginosa against which very few agents are effective (Morita et al., 2014; Poole, 2014). This so-called "antibiotic resistance crisis" has been compounded by the lagging in antibiotic discovery and development programs occurred in recent years, and is jeopardizing the essential role played by antibiotics in current medical practices (Rossolini et al., 2014). To combat this organism, it is very useful to understand its antimicrobial resistance mechanisms (see reviews such as Breidenstein et al., 2011; Poole, 2011; Morita et al., 2015).

The complete genome sequence of the multiresistant $P$. aeruginosa PA7 has been determined (Roy et al., 2010). The sequence of this strain, which exhibits resistance to fluoroquinolones (FQs), aminoglycosides, and various $\beta$-lactams but is susceptible to carbapenems (imipenem), includes typical FQ-resistance mutations in gyrA (Thr83Ile) and parC (Ser87Leu) (Roy et al., 2010). PA7 has additionally acquired a mutated aacA4 gene, the product of which $\left(\mathrm{AAC}\left(6^{\prime}\right)\right.$-II) endows the strain with aminoglycoside (gentamicin and tobramycin) resistance (Roy et al., 2010). This clinical isolate is amenable to the construction of gene knock-out mutations, thereby facilitating the analysis of molecular mechanisms of multidrug antimicrobial resistance in this isolate. Previously we showed that the effect of the modifying enzyme is enhanced by the MexXY resistance nodulation cell division (RND) -type multidrug efflux system, especially in the presence of divalent cations, providing high-level aminoglycoside resistance in P. aeruginosa (Morita et al., 2012b). This observation emphasizes the importance of the MexXY multidrug efflux system for aminoglycoside resistance in multidrug-resistant $P$. aeruginosa clinical isolates (Morita et al., 2012a).

Four members of the RND family of multidrug efflux systems, MexAB-OprM, MexCD-OprJ, MexEF-OprN, and MexXY have been implicated in $\mathrm{FQ}$ resistance in $P$. aeruginosa clinical isolates (Poole, 2011). As in other organisms, these RND family drug efflux transporters in $P$. aeruginosa operates as three-component pumps with an RND cytoplasmic membrane $(\mathrm{CM})$ protein $(\mathrm{MexB}, \mathrm{MexD}, \mathrm{MexF}$, and $\mathrm{MexY})$ linked to an outer membrane channel-forming protein (OprM, OprJ, and OprN) by a CM-tethered periplasmic protein (MexA, MexC, MexE, and MexX) (Poole, 2013). Intriguingly PA7 possesses the mexXY-oprA multidrug efflux operon of which oprA is missing in most P. aeruginosa strains (Roy et al., 2010). We showed that the MexXY can utilize either the OprA or OprM as an outer membrane component of the tripartite efflux pump (Morita et al., 2012b). Multidrug-resistant $P$. aeruginosa clinical isolates, 
including carbapenem-resistant $P$. aeruginosa, often have been reported to be MexXY and/or MexAB-OprM overproducers (e.g., Hocquet et al., 2007; Cabot et al., 2011; Fuste et al., 2013; Khuntayaporn et al., 2013; Vatcheva-Dobrevska et al., 2013). FQ efflux is mediated by the MexAB-OprM system constitutively produced at moderate levels in wild-type $P$. aeruginosa PAO1 (Morita et al., 2001; Poole, 2013). Expression of mexABoprM is controlled directly or indirectly by three repressors encoded by the mexR, nalC and nalD genes, with inactivating mutations in any of these resulting in increased mex $A B$ oprM expression (Poole, 2011, 2013). In addition, $n f x B$ and $n f x C$ mutants (which overproduce components of the MexCDOprJ and MexEF-OprN systems, respectively) are well-known as efflux-mediated FQ-resistant mutants of laboratory and clinical isolates of $P$. aeruginosa, although $n f x B$ mutations appear to be rare in clinical settings (Poole, 2011). $n f x C$ mutants also show increased resistance to carbapenems such as imipenem, not because MexEF-OprN accommodates these agents but because of a coordinated, MexT-dependent reduction of OprD production in such mutants (Poole, 2011, 2013). Hyperexpression of mexEF-orpN (and reduction in oprD expression) is also seen in laboratory isolates disrupted in the mexS gene, which encodes a putative oxidoreductase (a.k.a qrh) of unknown function (Sobel et al., 2005; Lamarche and Deziel, 2011). There are, however, no reports (to our knowledge) with experimental evidence of mexEFoprN-expressing clinical isolates harboring mutations in mexS (Poole, 2013), although some studies suggested possible mutation of mexS in $n f x C$-type clinical isolates (e.g., Llanes et al., 2011; Fournier et al., 2013). The incidence of MexXY overproducers among clinical isolates has shown to be linked to the use of ciprofloxacin (Hocquet et al., 2008) and MexXY overproducers have been often reported to be one of major phenotypes in multidrug resistant clinical isolates (Morita et al., 2012a; Poole, 2013). However, MexXY-expressing FQ-resistance or its contribution to FQ-resistance in clinical settings has not well been studied compared to the other three pumps. In this study, we used reverse genetics to investigate the role of efflux-mediated FQ resistance in multidrug-resistant $P$. aeruginosa clinical isolate PA7.

\section{MATERIALS AND METHODS BACTERIAL STRAINS, PLASMIDS, AND GROWTH CONDITIONS}

The bacterial strains and plasmids used in this study are listed in Table 1. Bacterial cells were grown in Luria (L) broth and on $\mathrm{L}$ agar $(1.5 \%)$ under aerobic conditions at $37^{\circ} \mathrm{C}$ as previously described, unless otherwise indicated, with antibiotics as necessary (Morita et al., 2010). Bacterial growth was quantified by measuring the optical density at $600 \mathrm{~nm}$ on an Ultrospec 2100 Pro Spectrophotometer (GE Healthcare Corp., Tokyo, Japan), unless otherwise indicated. The plasmids pEX18Tc (Hoang et al., 1998), pYM101 (Morita et al., 2010), and their derivatives were maintained and selected using medium supplemented with $2.5-10 \mu \mathrm{g}$ tetracycline $\mathrm{ml}^{-1}$ for E. coli or 50-150 $\mu \mathrm{g}$ tetracycline $\mathrm{ml}^{-1}$ for P. aeruginosa. The plasmids pUCP20T (Schweizer et al., 1996) and pFLP2 (Hoang et al., 1998) and their derivatives were maintained and selected using medium supplemented with $100 \mu \mathrm{g}$ ampicillin $\mathrm{ml}^{-1}$ for E. coli or $200 \mu \mathrm{g}$ carbenicillin $\mathrm{ml}^{-1}$ for P. aeruginosa.
Table 1 | Bacterial strains and plasmids.

\begin{tabular}{|c|c|c|}
\hline $\begin{array}{l}\text { Strains or } \\
\text { plasmids }\end{array}$ & Relevant characteristics & References \\
\hline \multicolumn{3}{|l|}{ E. COLI } \\
\hline $\begin{array}{l}\mathrm{DH} 5 \alpha \\
\text { (PAGUg 121) }\end{array}$ & For recombinant DNA manipulation & Morita et al., 2010 \\
\hline $\begin{array}{l}\text { KAM3 } \\
\left(\text { PAGU }^{9} 846\right)\end{array}$ & For recombinant DNA manipulation & Morita et al., 1998 \\
\hline $\begin{array}{l}\text { S17-1 } \\
\text { (PAGU9856) }\end{array}$ & For conjugational transfer & Morita et al., 2006 \\
\hline
\end{tabular}

\section{PAO1 (PAGU}

974)

PA7 (PAGU

Wild type

Morita et al., 2006

1498)

PAGUg 1565

PAGU9 1603

PAGUg 1748

PAGUg 1641

PAGU9 1751

PAGUg 1753

PAGUg 1756

Multi-resistant clinical isolate

Roy et al., 2010

PA7 $\triangle$ mexXY-oprA

PA7 $\triangle$ mexAB-oprM

PA7 $\triangle$ mexEF-oprN

PA7 $\triangle$ mexXY-oprA $\triangle$ mexAB-oprM

PA7 $\triangle$ mexXY-oprA $\triangle$ mexEF-oprN

PA7 $\triangle$ mexAB-oprM $\triangle$ mexEF-oprN

PA7 $\triangle$ mexXY-oprA $\triangle$ mexAB-oprM $\triangle$ mexEF-oprN

PAGU91793

PA7 $\triangle$ mexS

PAGU91789 PA7 $\triangle$ mexT

PAGU91867 PA7 mexS(V155A)

DSM 1128

(PAGU 1504)

Taxonomically PA7-related strain,

wild-type

K2153 (PAGU Clinical isolate

1741)

K2376

K2153 $\Delta$ mexS

Morita et al., 2012b

Morita et al., 2012b

This study

Morita et al., 2012b

This study

This study

This study

This study

This study

This study

Kiewitz and

Tummler, 2000

Sobel et al., 2003

(PAGU9 1834)

PAGUg 1850

K2153 $\Delta$ mexS attB::/ac/9-P

Fetar et al., 2011

PAGU9 1851

K2153 $\Delta$ mexS

attB::lac/9-P $P_{T 7}-\operatorname{mex} S_{P A 7}$

PAGU91844

K2153 $\Delta$ mexS

attB::/ac/9-P $\mathrm{P}_{T 7}-\operatorname{mex}_{D S M} 1128$

PAGU 1854

K2153 $\Delta$ mexS pUCP20T

PAGUg 1855

K2153 $\Delta$ mexS pUCP20T::mexS $S_{P A 7}$

K2153 $\Delta$ mexS

pUCP20T::mexS $S_{D S M} 1128$

PAGUg 1835

K2153 $\Delta$ mexT

PAGU 1836

K2153 $\Delta$ mexF

K2153 $\Delta$ mexS $\Delta$ mexT

This study

This study

This study

This study

This study

This study

Fetar et al., 2011

Fetar et al., 2011

K2942

(PAGU91837)
PAGU9 1852

K2153 $\Delta$ mexS $\Delta$ mexT

attB::lacla- $P_{T 7}$

PAGUg 1846

K2153 $\Delta$ mexS $\Delta$ mexT

attB::lac/9-P $P_{T 7}-$ mex $_{P A 7}$

PAGUg 1845

K2153 $\Delta$ mexS $\Delta$ mexT

attB::lac/9- $P_{T 7}-\operatorname{mex} T_{D S M} 1128$

\section{PLASMID}

Broad-host-range gene

replacement vector

pYM133

pEX18Tc:: $\Delta$ mexEF-oprN

pEX18Tc:: $\Delta \operatorname{mexS}$
Fetar et al., 2011

This study

This study

This study

Hoang et al., 1998

This study

This study 
Table 1 | Continued

\begin{tabular}{|c|c|c|}
\hline $\begin{array}{l}\text { Strains or } \\
\text { plasmids }\end{array}$ & Relevant characteristics & References \\
\hline pYM135 & pEX18Tc:: $\Delta \operatorname{mex} T$ & This study \\
\hline pYM136 & pEX18Tc:: $\Delta$ mexST & This study \\
\hline pYM137 & pEX18Tc::mexS $S_{P A 7}$ & This study \\
\hline pYM138 & pEX18Tc::mex $S_{P A 7}(V 155 A)$ & This study \\
\hline pYM101 & $\begin{array}{l}\text { P. aeruginosa chromosome } \\
\text { integration vector }\end{array}$ & Morita et al., 2010 \\
\hline pYM139 & pYM101::mex $S_{P A 7}$ & This study \\
\hline pYM140 & pYM101::mexS & This study \\
\hline pYM141 & pYM101::mexT $T_{P A 7}$ & This study \\
\hline pYM142 & pYM101::mexT $T_{D S M} 1128$ & This study \\
\hline pUCP20T & Broad-host-range cloning vector & $\begin{array}{l}\text { Schweizer et al., } \\
1996\end{array}$ \\
\hline pYM143 & pUCP20T::mexS $S_{P A 7}$ & This study \\
\hline pYM144 & pUCP20T::mexS $S_{D S M} 1128$ & This study \\
\hline pFLP2 & Flp recombinase-producing plasmid & Hoang et al., 1998 \\
\hline
\end{tabular}

\section{MOLECULAR BIOLOGY TECHNIQUES}

Plasmid DNA isolation from E. coli, DNA purification, measuring DNA concentration, DNA digestion with restriction enzymes, DNA dephosphorylation, DNA ligation, isolation of chromosomal DNA from $P$. aeruginosa, PCR conditions, nucleotide sequencing, competent cell preparation from E. coli, transformation of E. coli, and transfer of plasmids into $P$. aeruginosa via conjugation were performed as described previously (Morita et al., 2010), unless otherwise indicated. DNA sequences and amino acid sequences were analyzed through 2012 to 2013 with Pseudomonas Genome Database (Winsor et al., 2011), Basic Local Alignment Search Tool (BLAST) (Boratyn et al., 2013), SWISS-MODEL (Bordoli et al., 2009), Sorting Tolerant From Intolerant (SIFT) BLink (Kumar et al., 2009), and the software DNASIS Pro (Ver. 2.1; Hitachi, Japan).

\section{CLONING OF mexS AND mexT FROM P. AERUGINOSA}

mexS and mexT from $P$. aeruginosa (without endogenous promoters) were amplified by PCR using the primers listed in Table 2. The purified mexS PCR product, digested with BamHI and HindIII, was cloned into similarly digested, dephosphorylated pUCP20T and pYM101, yielding pUCP20T::mexS and pYM101::mexS. The purified mexT PCR product, digested with EcoRI and BamHI, was cloned into similarly digested, dephosphorylated pUCP20T and pYM101, yielding pUCP20T::mexT and pYM101::mexT.

\section{CONSTRUCTION OF IN-FRAME DELETION MUTANTS FROM P. AERUGINOSA}

In-frame deletion mutants of mexEF-oprN, mexS, and mexT from $P$. aeruginosa PA7 and its derivatives were constructed using the previously described sacB-based strategy (Morita et al., 2006, 2010). The plasmids and resulting $P$. aeruginosa mutants are listed in Table 1, while the primer pairs are listed in Table 2. The selection concentrations of tetracycline for the first homologous recombination event were adjusted to reflect the endogenous tetracycline MICs for the $P$. aeruginosa strains. These constructs were confirmed by colony PCR.

\section{CONSTRUCTION OF THE $\Phi$ CTX-BASED SITE-SPECIFIC INTEGRANTS IN P. AERUGINOSA}

For gene complementation experiments in P. aeruginosa, ФCTX phage-based site-specific integrants were constructed using the integration-proficient, tightly controlled expression vector pYM101 and associated techniques (conjugative transfer, gene replacement at the chromosomal $a t t B$ site, and curing of the unwanted plasmid backbone from the chromosome via the pFLP2-encoded Flp recombinase) as previously described (Morita et al., 2010).

\section{SITE-DIRECTED MUTAGENESIS OF mex $_{\text {PA7 }}$ IN THE CHROMOSOME OF P. AERUGINOSA PA7}

An amino acid substitution (Val155Ala) mutation was introduced into the mex $S_{P A 7}$ gene of the insert in the plasmid pUC20T::mexS $S_{P A 7}$. Site-directed mutagenesis within a target plasmid was carried out according to Geiser et al. (2001) and Morita et al. (2009). A 50- $\mu 1$ mixture consisting of $50 \mathrm{ng}$ of plasmid DNA, $0.25 \mu \mathrm{M}$ of each mutagenic primer pair (Table 2), $0.2 \mathrm{mM}$ each deoxynucleoside triphosphate, $1 \mathrm{mM} \mathrm{MgSO}_{4}, 2.5 \mathrm{U}$ of KOD Hot Start DNA polymerase -Plus- Ver.2 (TOYOBO Co. Ltd., Osaka, Japan), $1 \times$ KOD buffer, and 4.0\% ( $\mathrm{vol} / \mathrm{vol}$ ) dimethyl sulfoxide was heated to $94^{\circ} \mathrm{C}$ for $2 \mathrm{~min}$ followed by 18 cycles of $0.5 \mathrm{~min}$ at $94^{\circ} \mathrm{C}, 1 \mathrm{~min}$ at $60^{\circ} \mathrm{C}$, and $5 \mathrm{~min}$ at $68^{\circ} \mathrm{C}$. The resulting DNA products were purified as above, digested overnight with $10 \mathrm{U}$ DpnI (Roche Diagnostics K.K., Tokyo, Japan) to eliminate template plasmid), and used to transform E. coli DH5 $\alpha$. Plasmids were recovered from individual transformants, and the mexS insert was sequenced to identify plasmids bearing the desired mutation. The mexS insert carrying the mutation was digested with BamHI and HindIII and cloned into similarly digested and dephosphorylated pEX18Tc to yield pEX18Tc::mexS $S_{P A 7}(V 155 A)$. The mexS $S_{P A 7}(V 155 A)$ mutation was gene replaced onto the chromosome of $P$. aeruginosa PA7 using the previously described sacB-based strategy (Morita et al., 2009, 2012b).

\section{ANTIBIOTIC SUSCEPTIBILITY ASSAY}

The susceptibility of $P$. aeruginosa to antimicrobial agents in cation-adjusted Mueller-Hinton broth was assessed using the 2-fold serial microtiter broth dilution method described previously (Morita et al., 2012b). Minimal inhibitory concentrations (MICs) were defined as the lowest concentration of antibiotic resulting in visible inhibition of growth after $18 \mathrm{~h}$ of incubation at $37^{\circ} \mathrm{C}$. MICs for the $\Phi$ CTX-based site-specific integrants were determined in the presence of the inducer $5 \mathrm{mM}$ isopropyl- $\beta$-D1-thiogalactopyranoside (IPTG). The categorization in susceptible, intermediate, and resistant was performed according to the interpretive standards of the Clinical and Laboratory Standards Institute (CLSI) (Patel et al., 2011).

\section{ANTIMICROBIAL AGENTS}

Amikacin, ampicillin, carbenicillin, chloramphenicol, ciprofloxacin, norfloxacin, and tetracycline were purchased from Wako Pure Chemicals Industries, Ltd (Osaka, Japan). Moxifloxacin and levofloxacin were purchased from LKT 
Table 2 | Primers used in this study.

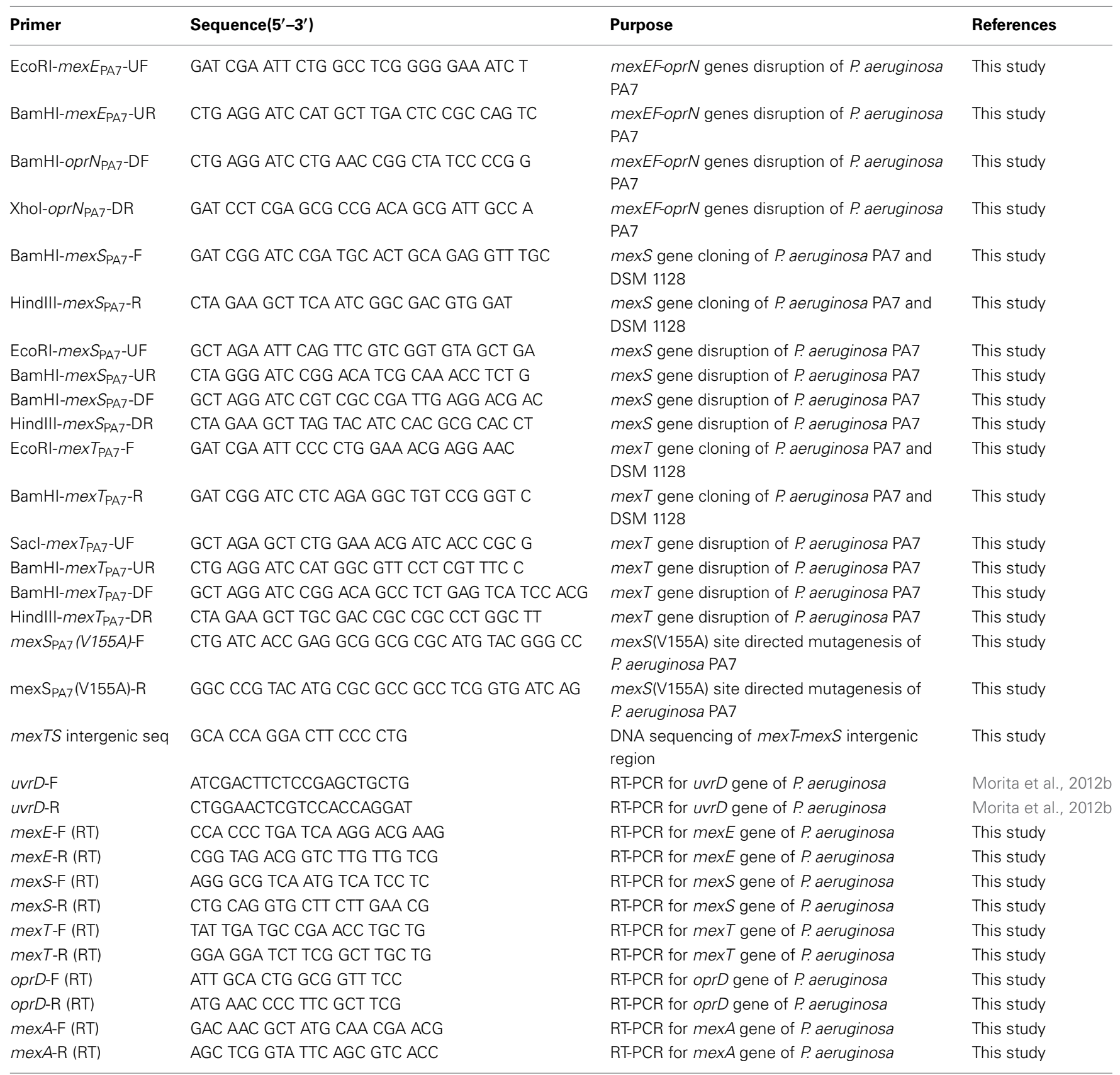

Laboratories, Inc. (St. Paul, MN, USA). Imipenem/cilastatin was purchased from Sandoz K.K. (Tokyo, Japan).

\section{REAL-TIME QUANTITATIVE REVERSE TRANSCRIPTASE (qRT)-PCR}

Overnight cultures of $P$. aeruginosa strains were diluted 1:100 in $10 \mathrm{ml}$, incubated with vigorous shaking at $37^{\circ} \mathrm{C}$ for $3-4 \mathrm{~h}$, and harvested. Total RNA was stabilized with the RNA Protect Bacteria Reagent (Qiagen) and isolated with the RNeasy Mini Kit (Qiagen). The RNA samples were further treated with RQ1 RNase-Free DNase (Promega) and purified by using the RNeasy Mini Kit (Qiagen). Real-time qRT-PCR was performed with primer pairs internal to uvrD, mexE, mexS, mexT, and oprD
(Table 2) using the One Step SYBR PrimeScript RT-PCR kit II (TaKaRa) in a Thermal Cycler Dice real-time system (TaKaRa). The transcript levels of the target gene in a given strain were normalized to levels of $u v r D$ and expressed as a ratio (fold change) to that observed in the parental PA7 strain. Gene expression values were calculated based on triplicate experiments.

\section{NUCLEOTIDE SEQUENCE ACCESSION NUMBER}

The nucleotide sequences of DNA regions containing mexT and mexS in $P$. aeruginosa DSM 1128 have been deposited in GenBank/EMBL/DDBJ with the accession number AB889539. 


\section{RESULTS \\ CONTRIBUTION OF RND MULTIDRUG EFFLUX SYSTEMS TO FO RESISTANCE IN P. AERUGINOSA PA7}

FQ resistance in $P$. aeruginosa PA7 was compared with the two FQ-susceptible strains, PAO1 (the standard laboratory strain) and DSM 1128 (a strain taxonomically related to PA7) (Morita et al., 2012b). The MICs of the FQs (ciprofloxacin, levofloxacin, moxifloxacin, and norfloxacin) for $P$. aeruginosa PA7, PAO1, and DSM 1128 are shown in Table 3. P. aeruginosa PA7 exhibited increased resistance (ca. 256-fold) to these FQs compared to $P$. aeruginosa PAO1 and DSM 1128. P. aeruginosa DSM 1128 showed the same level of resistance to the FQs as P. aeruginosa PAO1. Based on the interpretive standards of the CLSI (Patel et al., 2011), $P$. aeruginosa PA7 was considered highly resistant to these FQs. This observation is not surprising, because $P$. aeruginosa PA7 possesses typical target mutations, one in gyrA (Thr83Ile) and one in $\operatorname{parC}$ (Ser87Leu), well-known to be associated with FQ resistance (Lomovskaya et al., 1999). However, target mutations alone are not sufficient to explain high-level FQ resistance in $P$. aeruginosa; the additional effect of up-regulation of one of the four RND efflux pumps (MexXY-OprA, MexAB-OprM, MexCD-OprJ, or MexEF-OprN) is necessary (Lomovskaya et al., 1999; Bruchmann et al., 2013).

Previously we showed that the $\triangle$ mexXY-oprA $\triangle$ mexAB-oprM double mutant of PA7 was slightly more susceptible (ca. 2to 4-fold) to ciprofloxacin, while retaining high-level resistance $(\mathrm{MIC}=32 \mu \mathrm{g} / \mathrm{ml})$ to this antibiotic when compared to its parental strain (Morita et al., 2012b). That observation was inconsistent with data, derived via similar genetic analyses, in which a PAO1-derived $\triangle$ oprM gyrA parC triple mutant (i.e., a strain deleted for oprM and carrying the target mutations) exhibited increased susceptibility (64-fold) to levofloxacin compared to an isogenic MexAB-OprM overexpressing (nalB) mutant, while exhibiting an MIC $(0.5 \mu \mathrm{g} / \mathrm{ml})$ of levofloxacin similar to that of the wild-type PAO1 $(0.25 \mu \mathrm{g} / \mathrm{ml})$ (Lomovskaya et al., 1999). We noted that deletion of oprM in PAO1 was expected to inactivate any efflux pumps that require OprM as an outer membrane factor (e.g., MexAB-OprM, MexXY-OprM, MexVW-OprM) (Morita et al., 2001; Li et al., 2003). We therefore hypothesized that overexpression of other RND pumps such as MexCD-OprJ and MexEF-OprN was compensating for the effects of the mexABoprM mexXY-oprA double deficiency in PA7. As a first step in testing our hypothesis, we examined the primary sequences of the efflux system-encoding genes of PA7. Sequence analysis revealed the presence of mexCD-oprJ (PSPA7_0540- 0539- 0538) and mexEF-oprN (PSPA7_2745-2744-2743) homologs in P. aeruginosa PA7 (Roy et al., 2010), with predicted amino acid lengths (in PA7) and amino acid sequence identity and similarity [respectively; via BLAST of PA7 vs. PAO1-UW (Winsor et al., 2011)] as follows: MexC (387 aa; 88 and 95\%), MexD (1043 aa; 95 and 98\%,), OprJ (475 aa; 89 and 93\%); MexE (414 aa; 99 and 99\%), MexF (1062 aa; 99 and 99\%), and OprN (472 aa; 97 and 99\%). As a second step in testing our hypothesis, we examined the regulation of these genes in PA7. qRT-PCR showed that expression of mexE was increased (ca. 22-fold) in PA7 compared to the wild-type strain DSM 1128. This observation indicated that FQ resistance in $P$. aeruginosa can be mediated by overexpression of the mexEF-oprN operon, in addition to the previously reported overexpression of mexXY-oprA due to mutation of the local repressor gene (mexZ) (Morita et al., 2012b).

We also observed that a $\triangle$ mexXY-oprA $\triangle$ mexAB-oprM $\triangle$ mexEF-oprN triple mutant derived from PA7 exhibited increased susceptibility to FQs (32-128-fold) compared to an isogenic $\triangle$ mexXY-oprA $\triangle$ mexAB-oprM double mutant, while exhibiting only mild elevation of FQ MIC $(0.5-2 \mu \mathrm{g} / \mathrm{ml})$ compared to wild-type PAO1 and DSM $1128(0.125-1 \mu \mathrm{g} / \mathrm{ml})$ (Table 3). Moreover, the triple mutant was much more sensitive (512-fold) to chloramphenicol than the double mutant, a further indicator of MexEF-OprN-mediated resistance (Sobel et al., 2005) (Table 3). We excluded a role for overexpression of mexCD-oprJ in PA7, as judged by the susceptibilities of the mutant ( $\triangle m e x X Y$ oprA $\triangle$ mexAB-oprM $\triangle$ mexEF-oprN construct) to not only FQs but also to tetracycline and chloramphenicol, which are substrates of MexCD-OprJ (Poole et al., 1996).

To clarify the roles of MexXY-OprA, MexAB-OprM and MexEF-OprN multidrug efflux pumps in the FQ resistance of $P$. aeruginosa PA7, we constructed a series of deletion mutants of the three mex operons and examined their drug susceptibility

Table 3 | Contribution of three RND efflux pumps to antimicrobial resistance of $P$. aeruginosa PA7.

\begin{tabular}{|c|c|c|c|c|c|c|c|c|}
\hline \multirow[t]{2}{*}{ Strain } & \multirow[t]{2}{*}{ Genotype } & \multicolumn{7}{|c|}{$\operatorname{MIC}(\mu \mathrm{g} / \mathrm{ml})$ of } \\
\hline & & CIP & NOR & LVX & MXF & AMK & CHL & IPM \\
\hline PA7 (PAGU 1498) & Parent & 64 & 128 & 128 & 256 & 32 & 1024 & 2 \\
\hline PAGU9 1565 & PA7 $\Delta$ mexXY-oprA & 32 & 64 & 64 & 128 & 1 & 1024 & 2 \\
\hline PAGUg 1603 & PA7 $\triangle$ mexAB-oprM & 64 & 128 & 64 & 256 & 32 & 1024 & 2 \\
\hline PAGU91748 & PA7 $\Delta$ mexEF-oprN & 32 & 64 & 64 & 256 & 32 & 128 & 2 \\
\hline PAGUg 1641 & PA7 $\Delta$ mexXY-oprA $\Delta$ mexAB-oprM & 32 & 64 & 32 & 64 & 1 & 1024 & 2 \\
\hline PAGU91751 & PA7 $\Delta$ mexXY-oprA $\Delta$ mexEF-oprN & 8 & 16 & 8 & 32 & 1 & 256 & 2 \\
\hline PAGUg 1753 & PA7 $\Delta$ mexAB-oprM $\Delta$ mexEF-oprN & 32 & 64 & 32 & 128 & 32 & 16 & 2 \\
\hline PAGUg 1756 & PA7 $\Delta$ mexXY-oprA $\Delta$ mexAB-oprM $\Delta$ mexEF-oprN & 0.5 & 0.5 & 0.5 & 2 & 1 & 4 & 2 \\
\hline DSM 1128 (PAGU 1504) & Wild type & 0.125 & 0.25 & 0.25 & 1 & 0.5 & 32 & 1 \\
\hline PAO1 (PAGU 974) & Wild type & 0.125 & 0.25 & 0.25 & 1 & 0.5 & 32 & 1 \\
\hline
\end{tabular}

AMK, amikacin; CHL, chloramphenicol; CIP, ciprofloxacin; IPM, imipenem; LVX, levofloxacin; MXF, moxifloxacin; NOR, norfloxacin. 
profiles (Table 3). We observed that the $\triangle$ mexXY-oprA $\triangle$ mexEF$o p r N$ double deletion mutant was more susceptible (4-fold) than the $\triangle$ mexAB-oprM $\triangle$ mexXY-oprA double mutant and the $\triangle$ mexAB-oprM $\triangle$ mexEF-oprN double mutant (Table 3). These data suggested that the MexXY-OprA and MexEF-OprN systems make similar contributions to FQ resistance in $P$. aeruginosa PA7, each having an effect larger than that of MexAB-OprM. The apparent modest MexAB-OprM contribution to resistance of FQs and chloramphenicol in PA7 [PA7 $\triangle$ mexXY-oprA $\triangle$ mexEF-oprN vs. PA7 $\Delta$ mexXY-oprA $\Delta$ mexAB-oprM $\Delta$ mexEF-oprN (Table 3)] is typically observed in wild type $P$. aeruginosa such as PAO1 already shown in the previous results (e.g., PAO1 $\triangle$ mexXY vs. PAO1 $\Delta$ mexXY $\Delta$ mexAB-oprM) (e.g., Morita et al., 2001), implying that mexAB-oprM is expressed at moderate levels in PA7. We concluded that high-level FQ resistance in $P$. aerguinosa PA7 was due to overproduction of MexXY-OprA and MexEF-OprN, in addition to the typical target mutations.

\section{MOLECULAR MECHANISMS OF meXEF-oprN-UPREGULATION IN P. AERUGINOSA PA7}

The mexEF-oprN operon is quiescent in wild-type $P$. aeruginosa cells grown under standard laboratory conditions. In contrast, this operon is expressed in so-called $n f x C$ mutants and in mutants defective in the mexS gene (previously known as qrh), a locus that encodes a putative oxidoreductase of as yet unknown function (Poole, 2013). Expression of mexEF-oprN is regulated by a transcriptional activator, MexT, a LysR family regulator (Kohler et al., 1999; Poole, 2013). mexT occurs upstream of mexEF-oprN and downstream of mexS, the latter gene also positively regulated by MexT (Kohler et al., 1999). Unusually, many so-called wild type strains possess inactive MexT (e.g., 8 bp insertion in mexT present in some PAO1 strains) (Maseda et al., 2000; Poole, 2013). The induction of mexEF-oprN contributes to multidrug resistance, albeit against a rather narrow range of antimicrobials including FQs, trimethoprim, and chloramphenicol (Poole, 2013). The enhanced resistance to imipenem of $n f x C$ mutants or mexS deficient mutants results not from mexEF-oprN expression but the concomitant decrease in the level of outer membrane protein OprD (Poole, 2013), because OprD is an imipenem channel and serves as the primary route of entry of this antibiotic in $P$. aeruginosa (Trias and Nikaido, 1990). However, P. aeruginosa PA7 was reported to be susceptible to carbapenems, with MICs of 2 and $1 \mu \mathrm{g} / \mathrm{ml}$ for imipenem and meropenem, respectively (Roy et al.,
2010). This observation of carbapenem susceptibility is somewhat paradoxical, given that a typical $n f x C$ mutant or a mexS deficient mutant is expected to exhibit carbapenem resistance due to a coordinate, MexT-dependent reduction of OprD levels. We found that $P$. aeruginosa PA7 exhibited slight but reproducible elevation (2-fold) of resistance to imipenem compared to wild-type P. aeruginosa strains PAO1 and DSM 1128 (Table 4). Deletion of mexS in a PA7 background resulted in slightly increased expression of mexE (2-fold) and increased resistance to imipenem (4-fold) compared to PA7. Thus, PA7 appeared to exhibit susceptibility intermediate between that of wild type and a typical $n f x C$ mutant. Deletion of mexT in a PA7 background resulted in drastically ( $>200$-fold) decreased expression of mexE and mexS compared to PA7 (Table 4). Notably, expression of mexS in PA7 $\triangle m e x T$ was more than 96 -fold reduced compared to the wildtype DSM 1128 and PA7 mexS (V155A). This observation was consistent with a previous report (using $\beta$-galacotosidase reporter assays) indicating that mexS ( $q r h)$ is constitutively expressed at a moderate level (Kohler et al., 1999; Poole, 2013).

We therefore determined the nucleotide sequences (ca. $2.4 \mathrm{~kb}$ ) upstream of the mexEF-oprN operon in P. aeruginosa DSM 1128 (accession no. AB889539). This interval corresponds to the operon's cognate regulatory region, and includes the mexS and mexT loci. Comparison to the corresponding sequences from $P$. aeruginosa PA7 (data not shown) revealed that $\mathrm{MexS}_{\mathrm{PA}}$ is encoded as an $\mathrm{A} 155 \mathrm{~V}$ variant, and $\mathrm{MexT}_{\mathrm{PA} 7}$ as an $\mathrm{A} 256 \mathrm{~T}$ variant, compared to the DSM 1128 genome. In contrast, alanine-155 of MexS and alanine-256 of MexT are conserved among multiple laboratory and clinical strains, including PAO1, PA14, PAK, and K2153 (Sobel et al., 2005; Jin et al., 2011; Lamarche and Deziel, 2011). To test the function of the $m e x S_{P A 7}$ and $m e x T_{P A 7}$ loci, we amplified and cloned the individual mexS and mexT genes (without endogenous promoters) from $P$. aeruginosa PA7 and DSM 1128 , and expressed the relevant genes in P. aeruginosa K2153 $\Delta$ mexS (Fetar et al., 2011) for a mexS complementation test or in K2153 $\Delta$ mexS $\Delta$ mexT (Sobel et al., 2005; Fetar et al., 2011) for a mexT complementaton test (Table 5). [As demonstrated by other researchers, $P$. aeruginosa $\mathrm{K} 2153$ and its derivatives are useful for the analysis of mexEF-oprN-dependent antimicrobial resistance as regulated by the MexT activator and MexS function (Sobel et al., 2005; Fetar et al., 2011)]. Interestingly, introduction of mex $S_{D S M} 1128$ or mex $S_{P A 7}$ into the chromosome of K2153 $\Delta$ mexS did not provide complementation of $\Delta$ mexS (Table 5). These data

Table 4 | Relationship between antimicrobial resistance and mexS-mexT-mediated mexEF-oprN expression in $P$. aeruginosa.

\begin{tabular}{|c|c|c|c|c|c|c|c|}
\hline Strains & Genotype & \multicolumn{3}{|c|}{ MIC $(\mu \mathrm{g} / \mathrm{ml})$ of } & \multicolumn{3}{|c|}{ Relative mRNA level } \\
\hline PAGU91793 & PA7 $\Delta$ mexS & $>128$ & $>1024$ & 8 & 2.0 & nd & 0.84 \\
\hline PAGU9 1867 & PA7 mexS(V155A) & 64 & 256 & 1 & 0.053 & 0.57 & 0.74 \\
\hline PAGU9 1789 & PA7 $\Delta \operatorname{mex} T$ & 64 & 128 & 1 & $<0.005$ & $<0.005$ & nd \\
\hline
\end{tabular}

CHL, chloramphenicol; CIP, ciprofloxacin; IPM, imipenem; nd, not done. 
Table 5 | Functional characterization of $\operatorname{mex} S$ and $\operatorname{mex} T$ from P. aeruginosa PA7 and DSM 1128.

\begin{tabular}{|c|c|c|c|c|}
\hline \multirow[t]{2}{*}{ Strains } & \multirow[t]{2}{*}{ Genotype } & \multicolumn{3}{|c|}{$\operatorname{MIC}(\mu \mathrm{g} / \mathrm{ml})$} \\
\hline & & CIP & CHL & IPM \\
\hline K2153 (PAGU 1741) & Parent & 0.5 & 64 & 2 \\
\hline K2376 (PAGU91834) & $\Delta \operatorname{mexS}$ & 2 & 2048 & 8 \\
\hline PAGUg1850 & $\Delta \operatorname{mexS}$ attB::/ac/q-P $\mathrm{P}_{\mathrm{T} 7}$ & 2 & 2048 & 8 \\
\hline PAGU9 1851 & $\Delta$ mexS attB::/ac/q_P $P_{T 7}-m e x S_{P A 7}$ & 2 & 2048 & 8 \\
\hline PAGU91844 & $\begin{array}{l}\Delta \operatorname{mexS} \\
\text { attB::Iac/q-P } \\
T 7-\operatorname{mex} S_{D S M} 1128\end{array}$ & 2 & 1024 & 8 \\
\hline PAGU91854 & $\triangle \operatorname{mexS} \mathrm{pUCP} 20 \mathrm{~T}$ & 2 & 2048 & 8 \\
\hline PAGU9 1855 & $\Delta \operatorname{mexS}$ pUCP20T::mex $S_{P A 7}$ & 1 & 256 & 4 \\
\hline PAGU91856 & $\begin{array}{l}\Delta \operatorname{mexS} \\
\text { pUCP20T:: } \operatorname{mex} S_{D S M} 1128\end{array}$ & 0.5 & 64 & 2 \\
\hline K2942 (PAGU91837) & DmexS $\Delta \operatorname{mex} T$ & 0.5 & 64 & 2 \\
\hline PAGUg 1852 & $\Delta \operatorname{mexS} \Delta \operatorname{mex} T$ att $B:: / a c / q-P_{T 7}$ & 0.5 & 64 & 2 \\
\hline PAGU91846 & $\begin{array}{l}\Delta \operatorname{mexS} \Delta \operatorname{mex} T \\
\text { attB::lac/q-P } P_{\mathrm{T} 7}-\operatorname{mex} T_{P A 7}\end{array}$ & 2 & 2048 & 8 \\
\hline PAGU91845 & $\begin{array}{l}\Delta \operatorname{mex} S \Delta \operatorname{mex} T \\
\text { attB::/ac/ }{ }^{q}-P_{\mathrm{T} 7}-\operatorname{mex} T_{D S M} 1128\end{array}$ & 2 & 2048 & 8 \\
\hline
\end{tabular}

CHL, chloramphenicol; CIP, ciprofloxacin; IPM, imipenem.

$5 \mathrm{mM}$ IPTG was added to allow expression controlled by the $P_{T 7}$ promoter.

contrast previous instances in which we observed attB-site (i.e., single-copy) complementation for several other genes (Morita et al., 2010, 2012b). Presumably, in the present studies, failure to complement reflected insufficient mexS expression from the $\mathrm{P}_{\mathrm{T} 7}$ promoter in PAGU' 1844 , given that MexS $\mathrm{DSM}_{1128}$ is expected to be functional. Introduction of pUCP20T::mexS $S_{D S M} 1228$ into $P$. aeruginosa $\mathrm{K} 2153 \Delta$ mexS yielded MICs identical to those of the K2153 mexS ${ }^{+}$parent; introduction of pUCP20T::mex $S_{P A 7}$ yielded parent-like resistance to imipenem, but only partially restored resistance to ciprofloxacin and chloramphenicol (Table 5). Taken together, these results suggested that $\mathrm{MexS}_{\mathrm{PA}}$ provides reduced function compared to MexS DSM 1228. Additionally it appears that the high levels of mexS expression are required to overcome the $n f x C$-type antimicrobial resistance.

Slightly increased expression of mexS was observed in $P$. aeruginosa PA7 compared to P. aeruginosa DSM 1128 (ca. 2-fold), while expression levels of mexT in $P$. aeruginosa PA7 were similar to those in P. aeruginosa DSM 1128 (c.a. 0.9-fold) (Table 4). Introduction of mex $T_{D S M} 1128$ or $m e x T_{P A 7}$ into the chromosome of K2153 $\Delta$ mexS $\Delta$ mexT yielded the $n f x C$ phenotype (Table 5), suggesting that the A256T substitution in MexT is not a primary reason for overexpression of mexEF-oprN in $P$. aeruginosa PA7. Using site-specific mutagenesis, we altered the plasmidborne mex $S_{P A 7}$ locus to encode a V155A version of the protein and replaced the endogenous PA7 chromosomal locus with the mutated gene. The PA7 mexS $S_{P A 7}(V 155 A)$ strain showed decreased mexE expression (0.053-fold compared to that of the PA7 parent), a value similar to that seen in DSM 1128 (0.034-fold compared to that of PA7) (Table 4). The PA7 mexS $S_{P A 7}(V 155 A)$ strain also showed decreased MICs for chloramphenicol (0.25-fold) (Table 4). Taken together with the complementation experiments, these data strongly suggested that the A155V substitution in $\mathrm{MexS}_{\mathrm{PA7}}$ is the primary reason for increased expression of mexEF-oprN and increased antimicrobial resistance in the PA7 strain.

\section{DISCUSSION}

In this study, the multidrug-resistant clinical isolate PA7 was shown to exhibit increased expression of mexEF-oprN as well as mexXY-oprA. Although multidrug-resistant $P$. aeruginosa clinical isolates have often been reported to be MexXY overproducers (Morita et al., 2012a), clinical strains of $P$. aeruginosa overproducing MexEF-OprN and MexXY(-OprA) efflux pumps simultaneously have rarely been reported. In fact, PA7 was not a typical $n f x C$ mutant (i.e., a MexEF-OprN overproducer), and instead expressed intermediate levels of mexEF-oprN. We assume that simultaneous overproduction of MexEF-OprN and MexXY(OprA) impairs $P$. aeruginosa growth, based on our observation that our PA7 $\triangle$ mexS construct (i.e., a simultaneous overproducer of MexEF-OprN and MexXY-OprA compared to the PA7 parent) was unstable even on L agar plates: colonies of the construct exhibited a non-uniform phenotype during growth on plates (data not shown). This observation is consistent with the increased susceptibility to aminoglycosides previously observed in MexEF-OprN-overproducing $n f x C$ mutants, apparently owing to impairment of the MexXY system (Sobel et al., 2005).

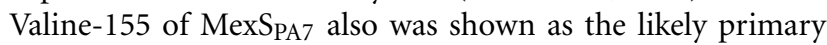
reason for increased production of MexEF-OprN in PA7. With the exception of mex $S_{P A 7}$, sequenced $P$. aeruginosa mexS genes (including those from PAO1, K2153, PA14, PAK, and DSM 1128) encode proteins with an alanine at residue 155 (Sobel et al., 2005; Jin et al., 2011; Lamarche and Deziel, 2011). The Ala155Val substitution is predicted by the SIFT algorithm (Kumar et al., 2009) not to affect the protein's function (data not shown). We hypothesize that MexS $\mathrm{S}_{\mathrm{PA}}$ retains function, albeit with decreased activity and/or altered regulation (e.g., allostery), compared with the other MexS orthologs. In fact, there were few differences among

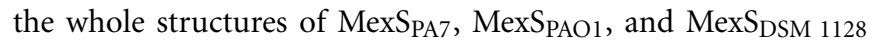
models developed by using the SWISS-MODEL program (data not shown) (Bordoli et al., 2009). MexS is a member of the cd08268: MDR2 family of the Conserved Domains Database (CDD) of the National Center for Biotechnology Information (NCBI) (Fargier et al., 2012) and alanine-155 corresponds to one of the putative $\mathrm{NAD}(\mathrm{P})$ binding sites featured in the MDR2 family.

In addition to antibiotic resistance, an $n f x C$-type mutation has been linked to reduced levels of homoserine lactone-dependent quorum sensing (QS) -regulated virulence factors, including pyocyanin, elastase, rhamnolipids, and Pseudomonas Quinolone Signal (PQS), and to reduced expression of type-III secretion system (TTSS) effector proteins (Kohler et al., 2001; Linares et al., 2005). QS is a cell-to-cell communication mechanism employing diffusible signal molecules (Jimenez et al., 2012), and the TTSS is a mechanism by which bacterial pathogens can deliver effectors directly into the cytoplasm of eukaryotic host cells (Hauser, 2009). We found that PA7 had reduced level of pyocyanin production, rhamnolipid production, and swarming activity compared to DSM 1128 and PAO1 (data not shown), consistent with the typical $n f x C$ mutant phenotype (Jin et al., 2011). However, PA7 


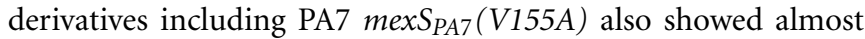
the same activities of the QS regulated virulence factors with PA7 (data not shown), which suggests that the impaired QSrelated phenotype is not derived from the $n f x C$-like phenotype in PA7. We presume that this lack of correlation reflects the absence from PA7 of TTSS-encoding genes and of the $m v f R$ ( $p q s R)$ gene, which is known to encode a LysR-type transcriptional regulator that modulates the expression of multiple QS-regulated virulence factors (Deziel et al., 2005; Roy et al., 2010). These deficiencies might be the source of the mexS mutation and increased mexEF-oprN expression under oxidative stress, sulfide stress, or nitrosative stress (Juhas et al., 2004; Fetar et al., 2011; Fargier et al., 2012).

\section{ACKNOWLEDGMENTS}

We thank Tadashi Kumazawa for his contribution. We thank Dr. Keith Poole (Queen's University, Canada) for providing the $P$. aeruginosa strains. This work was supported in part by a Grant-in-Aid for Young Scientists (B) (Kakenhi 23790106) and a Grant-in-Aid for Scientific Research (C) (Kakenhi 26460080) from the Japan Society for the Promotion of Science, and by a research grant from the Institute of Pharmaceutical Life Sciences, Aichi Gakuin University.

\section{REFERENCES}

Boratyn, G. M., Camacho, C., Cooper, P. S., Coulouris, G., Fong, A., and Ma, N. (2013). BLAST: a more efficient report with usability improvements. Nucleic Acids Res. 41, W29-W33. doi: 10.1093/nar/gkt282

Bordoli, L., Kiefer, F., Arnold, K., Benkert, P., Battey, J., and Schwede, T. (2009). Protein structure homology modeling using SWISS-MODEL workspace. Nat. Protoc. 4, 1-13. doi: 10.1038/nprot.2008.197

Breidenstein, E. B., De La Fuente-Nunez, C., and Hancock, R. E. (2011). Pseudomonas aeruginosa: all roads lead to resistance. Trends Microbiol. 19, 419-426. doi: 10.1016/j.tim.2011.04.005

Bruchmann, S., Dotsch, A., Nouri, B., Chaberny, I. F., and Haussler, S. (2013). Quantitative contributions of target alteration and decreased drug accumulation to Pseudomonas aeruginosa fluoroquinolone resistance. Antimicrob. Agents Chemother. 57, 1361-1368. doi: 10.1128/aac.01581-12

Cabot, G., Ocampo-Sosa, A. A., Tubau, F., Macia, M. D., Rodriguez, C., and Moya, B. (2011). Overexpression of AmpC and efflux pumps in Pseudomonas aeruginosa isolates from bloodstream infections: prevalence and impact on resistance in a Spanish multicenter study. Antimicrob. Agents Chemother. 55, 1906-1911. doi: 10.1128/aac.01645-10

Deziel, E., Gopalan, S., Tampakaki, A. P., Lepine, F., Padfield, K. E., and Saucier, M. (2005). The contribution of MvfR to Pseudomonas aeruginosa pathogenesis and quorum sensing circuitry regulation: multiple quorum sensing-regulated genes are modulated without affecting lasRI, rhlRI or the production of $\mathrm{N}$ acyl-L-homoserine lactones. Mol. Microbiol. 55, 998-1014. doi: 10.1111/j.13652958.2004.04448.x

Fargier, E., Mac Aogain, M., Mooij, M. J., Woods, D. F., Morrissey, J. P., and Dobson, A. D. (2012). MexT functions as a redox-responsive regulator modulating disulfide stress resistance in Pseudomonas aeruginosa. J. Bacteriol. 194, 3502-3511. doi: 10.1128/jb.06632-11

Fetar, H., Gilmour, C., Klinoski, R., Daigle, D. M., Dean, C. R., and Poole, K. (2011). mexEF-oprN multidrug efflux operon of Pseudomonas aeruginosa: regulation by the MexT activator in response to nitrosative stress and chloramphenicol. Antimicrob. Agents Chemother. 55, 508-514. doi: 10.1128/aac.00830-10

Fournier, D., Richardot, C., Muller, E., Robert-Nicoud, M., Llanes, C., and Plesiat, P. (2013). Complexity of resistance mechanisms to imipenem in intensive care unit strains of Pseudomonas aeruginosa. J. Antimicrob. Chemother. 68, 1772-1780. doi: $10.1093 /$ jac/dkt098

Fuste, E., Lopez-Jimenez, L., Segura, C., Gainza, E., Vinuesa, T., and Vinas, M. (2013). Carbapenem-resistance mechanisms of multidrug-resistant
Pseudomonas aeruginosa. J. Med. Microbiol. 62, 1317-1325. doi: 10.1099/jmm.0.058354-0

Geiser, M., Cebe, R., Drewello, D., and Schmitz, R. (2001). Integration of PCR fragments at any specific site within cloning vectors without the use of restriction enzymes and DNA ligase. Biotechniques 31, 88-92.

Gellatly, S. L., and Hancock, R. E. (2013). Pseudomonas aeruginosa: new insights into pathogenesis and host defenses. Pathog. Dis. 67, 159-173. doi: 10.1111/2049-632x.12033

Hauser, A. R. (2009). The type III secretion system of Pseudomonas aeruginosa: infection by injection. Nat. Rev. Microbiol. 7, 654-665. doi: 10.1038/nrmicro2199

Hoang, T. T., Karkhoff-Schweizer, R. R., Kutchma, A. J., and Schweizer, H. P. (1998). A broad-host-range Flp-FRT recombination system for site-specific excision of chromosomally-located DNA sequences: application for isolation of unmarked Pseudomonas aeruginosa mutants. Gene 212, 77-86.

Hocquet, D., Berthelot, P., Roussel-Delvallez, M., Favre, R., Jeannot, K., and Bajolet, O. (2007). Pseudomonas aeruginosa may accumulate drug resistance mechanisms without losing its ability to cause bloodstream infections. Antimicrob. Agents Chemother. 51, 3531-3536. doi: 10.1128/aac.00503-07

Hocquet, D., Muller, A., Blanc, K., Plesiat, P., Talon, D., Monnet, D. L., et al. (2008). Relationship between antibiotic use and incidence of MexXY-OprM overproducers among clinical isolates of Pseudomonas aeruginosa. Antimicrob. Agents Chemother. 52, 1173-1175. doi: 10.1128/aac.01212-07

Jimenez, P. N., Koch, G., Thompson, J. A., Xavier, K. B., Cool, R. H., and Quax, W. J. (2012). The multiple signaling systems regulating virulence in Pseudomonas aeruginosa. Microbiol. Mol. Biol. Rev. 76, 46-65. doi: 10.1128/mmbr.05007-11

Jin, Y., Yang, H., Qiao, M., and Jin, S. (2011). MexT regulates the type III secretion system through MexS and PtrC in Pseudomonas aeruginosa. J. Bacteriol. 193, 399-410. doi: 10.1128/jb.01079-10

Juhas, M., Wiehlmann, L., Huber, B., Jordan, D., Lauber, J., and Salunkhe, P. (2004). Global regulation of quorum sensing and virulence by VqsR in Pseudomonas aeruginosa. Microbiology 150, 831-841. doi: 10.1099/mic.0.26906-0

Khuntayaporn, P., Montakantikul, P., Santanirand, P., Kiratisin, P., and Chomnawang, M. T. (2013). Molecular investigation of carbapenem resistance among multidrug-resistant Pseudomonas aeruginosa isolated clinically in Thailand. Microbiol. Immunol. 57, 170-178. doi: 10.1111/1348-0421.12021

Kiewitz, C., and Tummler, B. (2000). Sequence diversity of Pseudomonas aeruginosa: impact on population structure and genome evolution. J. Bacteriol. 182, 3125-3135. doi: 10.1128/JB.182.11.3125-3135.2000

Kohler, T., Epp, S. F., Curty, L. K., and Pechere, J. C. (1999). Characterization of MexT, the regulator of the MexE-MexF-OprN multidrug efflux system of Pseudomonas aeruginosa. J. Bacteriol. 181, 6300-6305.

Kohler, T., Van Delden, C., Curty, L. K., Hamzehpour, M. M., and Pechere, J. C. (2001). Overexpression of the MexEF-OprN multidrug efflux system affects cellto-cell signaling in Pseudomonas aeruginosa. J. Bacteriol. 183, 5213-5222. doi: 10.1128/JB.183.18.5213-5222.2001

Kumar, P., Henikoff, S., and Ng, P. C. (2009). Predicting the effects of coding nonsynonymous variants on protein function using the SIFT algorithm. Nat. Protoc. 4, 1073-1081. doi: 10.1038/nprot.2009.86

Lamarche, M. G., and Deziel, E. (2011). MexEF-OprN efflux pump exports the Pseudomonas quinolone signal (PQS) precursor HHQ (4-hydroxy-2heptylquinoline). PLoS ONE 6:e24310. doi: 10.1371/journal.pone.0024310

Li, Y., Mima, T., Komori, Y., Morita, Y., Kuroda, T., and Mizushima, T. (2003). A new member of the tripartite multidrug efflux pumps, MexVW-OprM, in Pseudomonas aeruginosa. J. Antimicrob. Chemother. 52, 572-575. doi: $10.1093 / \mathrm{jac} / \mathrm{dkg} 390$

Linares, J. F., Lopez, J. A., Camafeita, E., Albar, J. P., Rojo, F., and Martinez, J. L. (2005). Overexpression of the multidrug efflux pumps MexCD-OprJ and MexEF-OprN is associated with a reduction of type III secretion in Pseudomonas aeruginosa. J. Bacteriol. 187, 1384-1391. doi: 10.1128/jb.187.4.1384-13 91.2005

Llanes, C., Kohler, T., Patry, I., Dehecq, B., Van Delden, C., and Plesiat, P. (2011). Role of the MexEF-OprN efflux system in low-level resistance of Pseudomonas aeruginosa to ciprofloxacin. Antimicrob. Agents Chemother. 55, 5676-5684. doi: 10.1128/aac.00101-11

Lomovskaya, O., Lee, A., Hoshino, K., Ishida, H., Mistry, A., and Warren, M. S. (1999). Use of a genetic approach to evaluate the consequences of inhibition of efflux pumps in Pseudomonas aeruginosa. Antimicrob. Agents Chemother. 43, $1340-1346$ 
Maseda, H., Saito, K., Nakajima, A., and Nakae, T. (2000). Variation of the mexT gene, a regulator of the MexEF-oprN efflux pump expression in wild-type strains of Pseudomonas aeruginosa. FEMS Microbiol. Lett. 192, 107-112. doi: 10.1111/j.1574-6968.2000.tb09367.x

Morita, Y., Gilmour, C., Metcalf, D., and Poole, K. (2009). Translational control of the antibiotic inducibility of the PA5471 gene required for mexXY multidrug efflux gene expression in Pseudomonas aeruginosa. J. Bacteriol. 191, 4966-4975. doi: 10.1128/jb.00073-09

Morita, Y., Kimura, N., Mima, T., Mizushima, T., and Tsuchiya, T. (2001). Roles of MexXY- and MexAB-multidrug efflux pumps in intrinsic multidrug resistance of Pseudomonas aeruginosa PAO1. J. Gen. Appl. Microbiol. 47, 27-32. doi: 10.2323/jgam.47.27

Morita, Y., Kodama, K., Shiota, S., Mine, T., Kataoka, A., and Mizushima, T. (1998). NorM, a putative multidrug efflux protein, of Vibrio parahaemolyticus and its homolog in Escherichia coli. Antimicrob. Agents Chemother. 42, 1778-1782.

Morita, Y., Narita, S., Tomida, J., Tokuda, H., and Kawamura, Y. (2010). Application of an inducible system to engineer unmarked conditional mutants of essential genes of Pseudomonas aeruginosa. J. Microbiol. Methods 82, 205-213. doi: 10.1016/j.mimet.2010.06.001

Morita, Y., Sobel, M. L., and Poole, K. (2006). Antibiotic inducibility of the MexXY multidrug efflux system of Pseudomonas aeruginosa: involvement of the antibiotic-inducible PA5471 gene product. J. Bacteriol. 188, 1847-1855. doi: 10.1128/jb.188.5.1847-1855.2006

Morita, Y., Tomida, J., and Kawamura, Y. (2012a). MexXY multidrug efflux system of Pseudomonas aeruginosa. Front. Microbiol. 3:408. doi: 10.3389/fmicb.2012.00408

Morita, Y., Tomida, J., and Kawamura, Y. (2012b). Primary mechanisms mediating aminoglycoside resistance in the multidrug-resistant Pseudomonas aeruginosa clinical isolate PA7. Microbiology 158, 1071-1083. doi: 10.1099/mic.0.054320-0

Morita, Y., Tomida, J., and Kawamura, Y. (2014). Responses of Pseudomonas aeruginosa to antimicrobials. Front. Microbiol. 4:422. doi: 10.3389/fmicb.2013.00422

Morita, Y., Tomida, J., and Kawamura, Y. (2015). "Resistance and response to anti-pseudomonas agents and biocides," in Pseudomonas: New Aspects of Pseudomonas Biology, eds J. Ramos, J. B. Goldberg, and A. Filloux (New York, NY: Springer), 173-187.

Patel, J. B., Tenover, F. C., Turnidge, J. D., and Jorgensen, J. H. (2011). "Susceptibility test methods: dilution and disk diffusion methods," in Manual of Clinical Microbiology, 10th Edn, eds J. Versalovic, K. C. Carroll, G. Funke, J. H. Jorgensen, M. L. Landry, and D. W. Warnock (Washington, DC: ASM Press), 1122-1143.

Poole, K. (2011). Pseudomonas aeruginosa: resistance to the max. Front. Microbiol. 2:65. doi: 10.3389/fmicb.2011.00065

Poole, K. (2013). "Pseudomonas aeruginosa efflux pumps," in Microbial Efflux Pumps: Current Research, eds E. W. Yu, Q. Zhang, and M. H. Brown (Norfolk: Caiser Academic Press), 175-206.

Poole, K. (2014). Stress responses as determinants of antimicrobial resistance in Pseudomonas aeruginosa: multidrug efflux and more. Can. J. Microbiol. 60, 783-791. doi: 10.1139/cjm-2014-0666

Poole, K., Gotoh, N., Tsujimoto, H., Zhao, Q., Wada, A., and Yamasaki, T. (1996). Overexpression of the mexC-mexD-oprJ efflux operon in $n f \times B$-type multidrugresistant strains of Pseudomonas aeruginosa. Mol. Microbiol. 21, 713-724.
Rossolini, G. M., Arena, F., Pecile, P., and Pollini, S. (2014). Update on the antibiotic resistance crisis. Curr. Opin. Pharmacol. 18c, 56-60. doi: 10.1016/j.coph.2014.09.006

Roy, P. H., Tetu, S. G., Larouche, A., Elbourne, L., Tremblay, S., and Ren, Q. (2010). Complete genome sequence of the multiresistant taxonomic outlier Pseudomonas aeruginosa PA7. PLoS ONE 5:e8842. doi: 10.1371/journal.pone.0008842

Schweizer, H. P., Klassen, T. R., and Hoang, T. T. (1996). "Improved methods for gene analysis and expression in Pseudomonas," in Molecular Biology of Pseudomonads, eds T. Nakazawa, D. Haas, and S. Silver (Washington, DC: ASM Press), 229-237.

Sobel, M. L., McKay, G. A., and Poole, K. (2003). Contribution of the MexXY multidrug transporter to aminoglycoside resistance in Pseudomonas aeruginosa clinical isolates. Antimicrob. Agents Chemother. 47, 3202-3207. doi: 10.1128/AAC.47.10.3202-3207.2003

Sobel, M. L., Neshat, S., and Poole, K. (2005). Mutations in PA2491 (mexS) promote MexT-dependent mexEF-oprN expression and multidrug resistance in a clinical strain of Pseudomonas aeruginosa. J. Bacteriol. 187, 1246-1253. doi: 10.1128/jb.187.4.1246-1253.2005

Trias, J., and Nikaido, H. (1990). Protein D2 channel of the Pseudomonas aeruginosa outer membrane has a binding site for basic amino acids and peptides. J. Biol. Chem. 265, 15680-15684.

Vatcheva-Dobrevska, R., Mulet, X., Ivanov, I., Zamorano, L., Dobreva, E., and Velinov, T. (2013). Molecular epidemiology and multidrug resistance mechanisms of Pseudomonas aeruginosa isolates from Bulgarian hospitals. Microb. Drug Resist. 19, 355-361. doi: 10.1089/mdr.2013.0004

Winsor, G. L., Lam, D. K., Fleming, L., Lo, R., Whiteside, M. D., and Yu, N. Y. (2011). Pseudomonas Genome Database: improved comparative analysis and population genomics capability for Pseudomonas genomes. Nucleic Acids Res. 39, D596-D600. doi: 10.1093/nar/gkq869

Conflict of Interest Statement: The authors declare that the research was conducted in the absence of any commercial or financial relationships that could be construed as a potential conflict of interest.

Received: 27 October 2014; paper pending published: 21 November 2014; accepted: 05 January 2015; published online: 21 January 2015.

Citation: Morita Y, Tomida J and Kawamura Y (2015) Efflux-mediated fluoroquinolone resistance in the multidrug-resistant Pseudomonas aeruginosa clinical isolate PA7: identification of a novel MexS variant involved in upregulation of the mexEF-oprN multidrug efflux operon. Front. Microbiol. 6:8. doi: 10.3389/fmicb. 2015.00008

This article was submitted to Antimicrobials, Resistance and Chemotherapy, a section of the journal Frontiers in Microbiology.

Copyright (c) 2015 Morita, Tomida and Kawamura. This is an open-access article distributed under the terms of the Creative Commons Attribution License (CC BY). The use, distribution or reproduction in other forums is permitted, provided the original author(s) or licensor are credited and that the original publication in this journal is cited, in accordance with accepted academic practice. No use, distribution or reproduction is permitted which does not comply with these terms. 